\title{
Le mémorial régional de la Grande Guerre à
} Sainte-Anne-d'Auray

Monument de la commémoration de masse catholique (1921-1937)

Frédéric Le Moigne

\section{OpenEdition}

\section{Journals}

Édition électronique

URL : http://journals.openedition.org/abpo/536

DOI : 10.4000/abpo.536

ISBN : 978-2-7535-1504-8

ISSN : 2108-6443

\section{Éditeur}

Presses universitaires de Rennes

\section{Édition imprimée}

Date de publication : 30 décembre 2006

Pagination : 49-76

ISBN : 978-2-7535-0405-9

ISSN : 0399-0826

\section{Référence électronique}

Frédéric Le Moigne, "Le mémorial régional de la Grande Guerre à Sainte-Anne-d'Auray », Annales de Bretagne et des Pays de l'Ouest [En ligne], 113-4 | 2006, mis en ligne le 30 décembre 2008, consulté le 01 mai 2019. URL : http://journals.openedition.org/abpo/536 ; DOI : 10.4000/abpo.536 


\title{
Le mémorial régional de la Grande Guerre à Sainte-Anne-d'Auray

\author{
Monument de la commémoration \\ de masse catholique (1921-1937)
}

\author{
Frédéric LE MoIGne \\ Chercheur associé au CRBC - Université de Bretagne occidentale
}

L'historiographie qui s'est beaucoup intéressée à la commémoration républicaine de la Grande Guerre (en particulier au monument aux Morts municipal) en distinguant, selon les cas et les régions, sa démarcation, sa captation ${ }^{1}$, son association ou sa dimension religieuse ${ }^{2}$, a moins souligné le fait que la commémoration catholique s'est également imposée à la République dans les hauts lieux de la mémoire nationale construits par la mort de masse (Douaumont, Notre-Dame de Lorette, Dormans). Si ces sites qui mériteraient d'être mieux comparés permettent déjà de poser la question de la mainmise catholique ponctuelle sur le deuil public de la Grande Guerre, celui de Sainte-Anne-d'Auray tranche assurément le débat à l'échelle de la Bretagne. Le cadre du sanctuaire et du pèlerinage se situe à la jonction du collectif intéressant l'histoire institutionnelle et de l'individuel scruté par l'anthropologie religieuse. En liant ainsi les « cercles du deuil ${ }^{3}$ " dans le cadre d'une identité régionale et catholique forte, il semble bien apparaître comme un espace privilégié du « deuil fier ", pour reprendre l'expression de Raoul Girardet, caractérisant en partie la société française de ce temps.

\section{Genèse d'une captation catholique (1918-1921)}

L'Église a détourné à son profit un projet né en décembre 1918 dans l'esprit de Charles Chaussepied, architecte des monuments historiques de

1. Suivre ici la démonstration - certes italienne - d'Emilio GENTILE sur l'invention de la religion de la patrie grâce à la liturgie civile commémorative de la guerre dans La religion fasciste. La sacralisation de la politique dans l'Italie fasciste, Paris, Perrin, 2002.

2. Voir pour l'Ouest, la synthèse de Jacqueline SAINCLIVIER dans La Bretagne dans l'ombre de la III République (1880-1939), Rennes, Ouest-France, 2005, p. 423-430.

3. Stéphane Audoin-Rouzeau, Cinq deuils de guerre, Paris, Noésis, 2001. 
Quimper. Ce dernier avait songé au site de roc'h Trévezel, près de Brasparts, pour y édifier le monument départemental en souvenir des morts de la Grande Guerre. Ce choix d'une colline - par référence à celle de Barrès ? - reproduisait ainsi le lien du champ de bataille entre la nature et les combattants. La solitude prenante et sauvage de cette hauteur finistérienne des Monts d'Arrée, royaume ancestral de la mort, avait de plus la légitimité d'abriter une chapelle sous l'invocation de saint Michel, ce que le catholique Chaussepied, futur architecte du grand séminaire de Quimper, ne voulait négliger.

Les plans dessinés (en particulier ceux d'une crypte regroupant tous les noms des disparus finistériens par paroisse), approuvés par les instances du département et par $\mathrm{M}^{\mathrm{gr}}$ Duparc, restèrent suspendus à la décision - longtemps réticente et retardée - de l'État et de la commission des sites. L'initiative avait en tout cas réveillé la Fédération régionaliste de Bretagne relayée par plusieurs journalistes d'Ille-et-Vilaine qui, après avoir soutenu le projet Chaussepied et croyant à son échec définitif, mena une active campagne dans Le Nouvelliste de Bretagne à partir de décembre 1920 visant au choix d'un nouveau site toujours lié à la symbolique de la hauteur - Menez-Hom, Bel-Air près de Moncontour -, ou à des panoramas du littoral - pointe du Raz, cap Fréhel.

Le débat passionné sur le site augurait mal d'une prise de décision rapide. Cela favorisa l'évêché de Vannes qui avait un projet plus solide. Dès la campagne de presse de décembre, le chanoine Buléon, influent curé de la cathédrale et figure du mouvement catholique breton, avait en effet formulé le souhait de voir le sanctuaire de Sainte-Anne-d'Auray - dont il était, avec son confrère Le Garrec, l'historiographe - accueillir le monument régional. Son évêque $\mathrm{M}^{\mathrm{gr}}$ Gouraud lui emboîta rapidement le pas en convoquant début janvier le marquis de l'Estourbeillon pour qu'il plaide la cause morbihannaise au sein de la Fédération régionaliste et plus particulièrement auprès de l'abbé Bossard chargé, lors de la réunion d'un Comité élargi à Rennes le 28 décembre, de trouver le parrainage du monument. L'injonction hiérarchique fut rapidement entendue de ce dernier ${ }^{4}$, toutefois le prêtre restait fixé sur sa mission (il invita d'ailleurs Gouraud comme son métropolitain rennais Dubourg à entrer dans le comité de parrainage) et n'entendait pas arrêter la procédure démocratique du Comité.

Une séance avait été en effet fixée au 26 février 1921, qui rendit publique les noms des principaux parrains du monument d'ailleurs assez habilement répartis sous une bannière "Union sacrée " (les généraux Foch, Weygand, les écrivains Le Goffic, Le Braz, les prélats Dubourg et Duchesne, les hommes politiques de gauche, Guernier et Lemarié comme de droite, Jénouvrier et Guilloteaux). Le titre de secrétaire général échappait à l'abbé Bossard au profit du rédacteur du Salut de Saint-Malo, Louis Boivin, celuilà même qui avait lancé la campagne du Nouvelliste. Les régionalistes catho-

4. Arch. dioc. de Vannes, lettre du 21 janvier 1921 à $M^{\text {gr }}$ Gouraud. 
liques perdirent en influence comme le signalait subrepticement l'apparition d'un titre de projet - plus acceptable pour l'État et ses éventuelles subventions - « Monument aux enfants de la France ». En ce sens, Boivin commençait d'ailleurs à évoquer un projet urbain rennais pour mieux s'opposer à Sainte-Anne-d'Auray qui n'était pas son choix.

Sentant peut-être le vent tourner, $\mathrm{M}^{\text {gr }}$ Gouraud, aussi opportuniste qu'intégraliste, fit acte d'autorité. Après avoir reçu fin mars un formulaire d'adhésion au comité du monument, auquel il ne prit pas la peine de répondre, il rendit publique sa décision de "faire " à Sainte-Anne-d'Auray " un monument où toute la Bretagne pourra se rendre, entrer et prier $^{5}$. "Sans fournir trop de détails, l'évêque de Vannes donnait l'illusion à ses diocésains d'une initiative mûrement réfléchie en l'inscrivant comme l'accomplissement du vœu, formulé durant la guerre, de construction d'un monument en remerciement de la protection de Jeanne d'Arc et du Sacré-Cœur. Cette assurance, notamment financière puisque les fidèles du Morbihan avaient déjà commencé à adresser des dons, et qui était peut-être destinée à ses frères en épiscopat de la province mis devant le fait accompli, était révélatrice de l'audace de Gouraud. Trois jours plus tard, le prélat recevait une lettre de Chaussepied faisant acte de candidature pour Sainte-Anne-d'Auray. L'architecte, déçu par l'échec de son projet finistérien et éloigné des initiatives rennaises, se recommandait de la marquise de Polignac et de son évêque Duparc. Cet empressement faisait cependant contraste avec l'attentisme choisi par les chefs des autres diocèses bretons. Ceux-ci patientèrent en effet jusqu'à l'été pour se réunir sur le sujet à Quimper. Aucun des évêques ne s'est opposé au choix de Sainte-Anne mais chacun avait un motif qui retenait encore une adhésion entière.

$\mathrm{M}^{\mathrm{gr}}$ Duparc, dont l'origine morbihannaise et les liens avec le sanctuaire étaient connus, ne voulait pas donner l'impression à ses diocésains d'avoir lâché le projet finistérien. Deux indices le prouvent. Durant l'hiver 1921, lorsque Gouraud lui avait révélé son projet, l'évêque de Quimper n'avait pas caché son " embarras " en affirmant qu'il était prêt à s'associer aux deux monuments puisqu'il nourrissait encore quelques espoirs quant à l'acceptation par l'État du site des monts d'Arrée. Par ailleurs, dans son grand discours prononcé lors de l'inauguration du mémorial en 1932, il réserva une place de choix au projet initial de Brasparts en l'associant, dans une envolée sur l'archange saint Michel, à la " prière du guetteur " de Jean-Pierre Calloc'h pour mieux souligner : "Les évêques bretons, en majorité, préférèrent confier à Sainte Anne la mémoire de nos morts. Ils eurent raison. La Basilique de Sainte Anne est le cœur de la Bretagne ${ }^{6}$." $\mathrm{M}^{\mathrm{gr}}$ Charost, coadjuteur rennais du déclinant $\mathrm{M}^{\mathrm{gr}}$ Dubourg ${ }^{7}$, trouva à redire non sur le fond - il ne semblait pas être très lié au comité rennais - mais

5. Semaine religieuse du diocèse de Vannes, 9 avril 1921, n 15, p. 233.

6. Ibidem, 30 juillet $1932, \mathrm{n}^{\circ} 31$, p. 501.

7. Incapable de se concentrer et de se déplacer durant le premier semestre 1921, il meurt le 21 septembre. 
sur la forme de l'initiative de Gouraud, à ses yeux trop personnelle et trop morbihannaise. Il l'exprima de manière détournée à l'intéressé en réservant son soutien "à la condition formelle que le monument n'aura pas un comité proprement diocésain; qu'il sera érigé par les soins et la coopération de tous les Évêques de la province, et que cela sera indiqué dans les feuilles publiques et sur le monument ${ }^{8}$ ". Le futur métropolitain de Bretagne fit d'ailleurs retarder la déclaration collective pour prendre le temps de l'étudier en juillet. Enfin, dernier prélat (puisque l'évêque de Nantes $\mathrm{M}^{\mathrm{gr}}$ Le Fer de La Motte ne s'est pas impliqué sinon pour associer formellement son diocèse), $\mathrm{M}^{\mathrm{gr}}$ Morelle a été le plus hésitant. Lors de la réunion de Quimper, l'évêque de Saint-Brieuc avait en effet réservé son soutien. Le prélat, précisément affaibli par un manque de considération des milieux régionalistes, craignait une situation de concurrence et un " conflit d'opinion " avec le comité rennais comme il le redit dans un courrier du 10 août à $\mathrm{M}^{\mathrm{gr}}$ Gouraud. Ce dernier préféra certainement retenir l'appui formel exprimé par son voisin pour publier sa déclaration rédigée depuis deux mois et enfin suivie des cinq noms de la hiérarchie catholique bretonne.

Ce texte, lu dans toutes les églises de Bretagne et qui en appelait pour la première fois à la générosité des fidèles pour pouvoir inaugurer sur un terrain privé attenant au sanctuaire le monument lors des festivités du $3^{\mathrm{e}}$ centenaire des apparitions de sainte Anne (1924 ou 1925), ne s'attardait pas davantage sur les modalités de l'entreprise. Par contre, l'esprit du projet était soigneusement exposé et ne cherchait nullement à gommer son caractère intégraliste. Puisque l'on construisait partout des monuments à la gloire des morts, l'Église voulait " avoir ses monuments à elle, à l'ombre de ses principaux sanctuaires ». Elle se devait en effet d'entretenir les pèlerinages de reconnaissance constatés depuis la fin de la guerre ${ }^{9}$ à Paray-le-Monial, Montmartre, Pontmain, et surtout à Lourdes qui servaient de référence à Gouraud, négligeant ainsi les nouveaux " sanctuaires " du champ de bataille (Douaumont, Notre-Dame de Lorette). Sainte-Anne-d'Auray, centre actif de prières et à ce titre désigné comme lieu de sépulture symbolique rêvé des combattants bretons, allait donc accueillir le monument qui développerait le pèlerinage et multiplierait les intentions pour les disparus.

La construction sanctuarisée de Gouraud confiée désormais à un comité réunissant les évêques et leurs représentants exclusivement ecclésiastiques (Abgrall, Buléon...) ne faisait aucune référence au comité rennais pour mieux s'en séparer, d'autant plus que celui-ci n'abandonnait pas facilement la partie. Il fallut en effet la convocation en octobre de Boivin par $\mathrm{M}^{\mathrm{gr}}$ Charost pour qu'il jette l'éponge. Le prix de sa renonciation, à savoir un article dans Le Nouvelliste ${ }^{10}$, se révéla un document assez embarrassant pour l'archevêque de Rennes puisque le publiciste ne cacha pas son amer-

8. Arch. dioc. de Vannes, lettre du 29 juin 1921.

9. Après les pèlerinages individuels du temps de guerre, Sainte-Anne d'Auray avait connu ces pèlerinages collectifs d'action de grâce à partir de mars 1919 .

10. Vendredi 7 octobre $1921, \mathrm{n}^{\circ} 280$ 
tume d'avoir été écarté et réaffirma une dernière fois, dans le quotidien conservateur, la légitimité de son projet politique qui, tout en gardant une identité catholique formelle (son monument devait avoir une croix, une flèche aérienne et une crypte), se voulait ouvert à tous les Bretons "de toutes opinions politiques et de toutes religions ". Force est cependant de constater que la conscience régionale politique n'était pas aussi prononcée que celle de l'Église, et cette atonie républicaine - qui ne peut être analysée ici - ne saurait être imputée à la seule Loire-Inférieure. Les pressions ultérieures qu'André Maginot dit avoir subies pour stopper le projet catholique n'ont donc peut-être pas été très sérieuses. En régime de Séparation qui élargit l'autonomie du pré-carré catholique, eussent-elles d'ailleurs été opérantes?

Au lendemain de l'article du Nouvelliste, les journaux annonçaient le début du concours d'architectes auquel l'Église cherchait à donner le maximum de publicité et de crédibilité pour lancer son projet. Une centaine de courriers d'information ont ainsi été expédiés de l'évêché de Vannes. Gouraud s'est appuyé sur son ancien élève de l'externat des Enfants nantais, l'architecte René Ménard, pour élaborer la procédure de désignation. Ce dernier - qui en profitait pour déclarer au prélat sa candidature... - l'a conçue à la fin août en copiant le concours contemporain du monument aux Morts de Versailles, jusqu'au prix de la construction fixée à 400000 francs. Ce souci de respecter la procédure officielle des concours publics - qui rompait avec la marque intégraliste manifestée jusque-là - se poursuivit avec le choix de deux cabinets parisiens reconnus pour la bonification du règlement et leur participation au jury. La Société des architectes diplômés par le gouvernement n'obtint cependant pas gain de cause sur l'ouverture du concours aux non Bretons mais fut mieux entendue de la hiérarchie sur l'augmentation des primes aux lauréats.

Sur quel dossier pouvaient s'appuyer les candidats pour élaborer leur projet? Outre le plan établi par le chanoine Pouëzat, supérieur de la basilique de Sainte-Anne, qui prouve que le mémorial a servi de prétexte à une extension vers les terrains du petit séminaire de l'espace de rassemblement traditionnel trop proche de la basilique et de la rue (et à un remodelage routier envisagé alors pour couper les rues à angle droit dangereuses pour la circulation mais qui n'aboutit pas), ils se voyaient adresser pour inspiration la lettre collective des évêques ainsi que le texte du comité d'édification :

"Le monument comprendra une sorte de chapelle funéraire avec autels, où seront inscrits les noms des paroisses des 5 diocèses bretons et le nombre des morts de chaque paroisse. On devra trouver, au-dessus ou en avant, sous la forme de loggia ou d'esplanade couverte d'environ $150 \mathrm{~m}^{2}$, un lieu de culte public, où pourront se célébrer, au regard de la foule, les cérémonies extérieures du pèlerinage de Sainte Anne, comme cela se fait maintenant à la Scala Sancta.» 


\section{Un démarrage laborieux (1922-1924)}

Le jury - uniquement ecclésiastique et technicien ${ }^{11}$ - présidé par l'architecte parisien Alexandre Marcel se réunit à Sainte-Anne-d'Auray le 16 janvier 1922 et retint quatre esquisses sur la quinzaine en compétition. René Guillaume de Lorient, Lionel Heuzé de Morlaix, René Ménard de Nantes et Pierre Regnault de Paris ${ }^{12}$ avaient ensuite exactement trois mois pour fournir des plans plus précis. Le 16 avril, Ménard remporta le concours. Au vu de ses liens avec Gouraud, de son profil d'architecte d'églises avec une étude familiale liée à l'évêché de Nantes, et de l'impression faite sur le jury dès le premier degré du concours avec un projet au titre programmatique apprécié - «Catholiques et Bretons toujours "-, qui misait sur un monument élancé de plein centre en granit, le résultat n'était guère surprenant. Il semble cependant que sa confortable avance ait fondu devant le deuxième jury. Celui-ci lui reprocha le caractère massif de sa nouvelle esquisse de mausolée et sa difficulté à aménager l'esplanade (suggestion d'une clôture monumentale pour marquer l'entrée depuis la fontaine sur la pelouse du chemin de croix). Il en venait même à remettre en cause son projet de crypte au profit de l'aménagement d'une clôture de 80 mètres de diamètre appelée Campo Santo entourant le monument comprenant un portique bas et les cinq chapelles des diocèses bretons; ce qui avait pour effet d'abaisser le mausolée. Cela exprimait un satisfecit appuyé au projet " Dolmen " de Guillaume arrivé second qui avait parié sur cet élément.

Ménard confia à son protecteur $\mathrm{M}^{\mathrm{gr}}$ Gouraud que son premier projet était meilleur, mais qu'il avait voulu l'amplifier, notamment en hauteur, pour résister à la concurrence ${ }^{13}$. L'architecture cachait assurément la question du financement qui commença à se poser avec acuité en ce début d'année 1922. Si les évêques avaient en effet rapidement tourné la page des 400000 francs, ils espérèrent ensuite ne pas dépasser 600000 francs (hors frais de terrassement) pour des raisons que l'on verra plus loin. On peut penser que Ménard, parfaitement renseigné sur ses attentes hiérarchiques et ayant bien lu les recommandations du jury après la première sélection demandant aux candidats de donner un plan d'ensemble " conçu de manière à pouvoir être exécuté en parties utilisables ", ne pouvait guère s'intéresser à l'enceinte puisque son projet de monument atteignait déjà

11. 5 représentants des évêchés (Abgrall pour Quimper, Bouchaud pour Nantes, Lecoqu pour Saint-Brieuc, Pouëzat pour Vannes, Prost pour Rennes) et 5 architectes. Ménard avait pourtant conseillé la présence de régionalistes et d'artistes bretons. Ces derniers ont certainement été écartés à cause du comité rennais.

12. Liste d'architectes rendue publique non par l'organe diocésain de Vannes mais par la Semaine religieuse de Quimper et Léon, 26 mai 1922, $\mathrm{n}^{\circ} 21$, p. 314. Sur ce milieu lire Daniel LE CouËDIC, Les Architectes et l'idée bretonne 1901-1945, Rennes, Société d'histoire et d'architecture de Bretagne, 1995, p. 627-630.

13. Arch. dioc. de Vannes, lettre du 25 mai 1922 adressée au secrétaire de Gouraud, le vicaire général Thubé. Celui-ci, d'origine nantaise, était lié à Ménard. Son père fut un des premiers souscripteurs du monument avec un don de 10000 francs. 
770000 francs $^{14}$. Son concurrent, Pierre Regnault, qui finit troisième avec le projet "Reconnaissance d'un combattant ", avait ainsi compromis ses chances en proposant un programme de 1,7 million de francs. Il faut dire que l'architecte avait prévu l'aménagement grandiose d'un « jardin du monument " en le délimitant notamment par des fossés d'eau captée de l'étang tout proche.

Ce même Regnault, contrairement au dernier candidat Lionel Heuzé 15 qui a accepté le verdict sur son projet "Crux " de 700000 francs d'autant plus facilement qu'il estimait le gagnant et n'avait pas bien pu préparer le concours à cause d'une longue grippe et d'un manque de personnel à Morlaix ${ }^{16}$, reçut très mal les résultats. Non seulement il fit le voyage jusqu'à Nantes pour déclarer à Ménard que le jugement du comité était « idiot " mais il publia dans Le Nouvelliste du 21 mai - décidément habitué à gêner le Comité de Sainte-Anne - un article extraordinaire. Le texte annoncé par le titre « Le monument commémoratif qui sera érigé au centre du pèlerinage de Sainte-Anne à la mémoire des 250000 Bretons tombés pendant la grande guerre " se voulait parfaitement renseigné et offrait aux lecteurs tous les détails qu'ils étaient en droit d'attendre sur l'emplacement et les attentes du jury. Seulement, il n'était que la copie intégrale du projet Regnault jusqu'à la reproduction en " une " de son dessin de monument écarté...

Le monument Ménard fut quant à lui dessiné durant l'été 1922 pour être diffusé sous forme de cartes postales (Figure 1) de l'éditeur nantais Chantreau au profit du comité d'exécution. Priorité était en effet donnée à la matérialisation du projet pour motiver les souscripteurs (comme plus tard les cartes postales photographiques). L'échec de la première quête régionale du 6 novembre 1921, pourtant inscrite dans la temporalité du souvenir, était principalement à rechercher dans ce blocage des fidèles « attendant de voir " avant de donner. Si les diocésains du Finistère, depuis plus longtemps sensibilisés que les autres par ce projet de monument régional et motivés par $\mathrm{M}^{\mathrm{gr}}$ Duparc, avaient offert 67000 francs, ceux des Côtes-duNord en étaient restés à 25500 francs (ce qui constituait le maximum de l'effort consenti par $\mathrm{M}^{\mathrm{gr}}$ Morelle, démoralisé par le coût du monument et attendant de mobiliser ses fidèles pour la construction d'un grand séminaire). Surtout, Nantes n'avait donné que 18000 francs et Rennes 13800, soit à peine plus que l'offrande du diocèse de Nancy (aidé par celui de Vannes durant la guerre) - 10000 francs, ou la souscription de René Bolloré - 5000 francs ${ }^{17}$.

Charost dut une explication à Gouraud. Il chargea son prédécesseur Dubourg, accusé d'avoir laissé s'assoupir depuis la guerre les générosités

14. Chiffre cité par Christian CHAUdRé, Le Mémorial 1914-1918 de Sainte-Anne d'Auray. Images d'une construction, Vannes, Archives départementales, 1998, p. 10.

15. Ce dernier fut l'architecte de la chapelle mémorial Notre-Dame-des-Anges étudiée par Yvon Tranvouez. Lire " La mémoire d'un bombardement britannique : Notre-Damedes-Anges (Morlaix, 1943-2003) ", Annales de Bretagne et des Pays de l'Ouest, t. 111, janvier 2004, p. 127-154.

16. Arch. de la basilique de Sainte-Anne d'Auray, lettre à Ménard du 26 mai 1922.

17. Annales du pèlerinage de Sainte-Anne d'Auray, $1^{\mathrm{er}}$ janvier $1923, \mathrm{n}^{\circ} 4$, p. 15. 
du diocèse, ce qui rendait prioritaires les quêtes pour les séminaires et les écoles. Très sollicités, ses diocésains éprouvaient des difficultés à s'identifier au projet catholique régional, à l'heure où les communes « rivalisaient un peu de gloriole entre elles " en construisant leurs monuments aux Morts. Gouraud, plus allergique encore à la liturgie commémorative républicaine ${ }^{18}$ qui, contrairement à son voisin, ne lui avait pas décerné de Légion d'honneur, dut être rasséréné par l'expression ainsi que les perspectives d'avenir optimistes esquissées pour le monument par le primat de Bretagne. Celles-ci lui étaient bien nécessaires et l'on perçoit à ce moment de la chronologie combien le Monument devait être imposé puisque Sainte-Anned'Auray ne pouvait prétendre au statut de " pèlerinage commun à tous les Bretons ${ }^{19}$ ".

$\mathrm{M}^{\mathrm{gr}}$ Gouraud - dont la santé était déclinante - a alors perçu qu'il ne verrait pas l'aboutissement de son projet. Le terrassement confié à l'entrepreneur vannetais Huchet, recommandé par l'architecte Caubert et préféré à André, candidat de Ménard, s'est en effet révélé long et coûteux. Les travaux commencés en octobre 1922 ont duré dix mois pour seulement parvenir à creuser 8 puits de 8 à 12 mètres. Le caractère marécageux du site a nécessité une lutte permanente contre les infiltrations d'eau et de sable, contraignant les ouvriers à une véritable course contre la montre en travaillant jour et nuit dans les dernières semaines.

Ces puits sans fond ont logiquement nourri la rumeur de sommes astronomiques perdues par l'Église; ce qui la contraignit à améliorer sa campagne d'informations sur l'avancée des travaux et le financement. La suspicion généralisée sur le choix du site - et qui eut des traces au moins jusqu'en 1926 - n'a pas aidé l'entrepreneur et l'architecte, réveillant contre ce dernier les rancours du concours. Voilà pourquoi les Annales du pèlerinage parlèrent, en pensant en particulier aux préoccupations du chanoine Pouëzat des " difficultés matérielles " et même " morales ${ }^{20}$ " de l'entreprise. Ce dernier fut de fait remplacé en 1926 par le chanoine Quelven, professeur de mathématiques à Saint-Louis de Lorient et ancien officier télégraphiste.

Les dons eux-mêmes n'ont guère progressé durant cette période, comme le prouve le peu de succès de la campagne enregistrant, contre l'offrande élevée de 150 francs, les noms des disparus avec promesse d'inscription sur le mur d'enceinte du futur chemin de croix. Cette extension de la pratique paroissiale, lancée en novembre 1922, n'avait intéressé que 4 familles en janvier 1923 et seulement 37 en juillet 1925. Il faut dire que les organisateurs, concentrés sur les fondations et les assises du monument central, ne pou-

18. On retrouve les réticences repérées localement par Didier Guyvarc'h et Patrick Gourlay. De ce dernier lire "Un exemple de mémoire " disputé " : les 2 monuments aux morts de Ploujean ", Annales de Bretagne et des Pays de l'Ouest, tome 109, 2002, n 1, p. $129-145$.

19. Michel Lagrée faisait naturellement référence à Lourdes, plus fédérateur, voir Religion et cultures en Bretagne 1850-1950, Paris, Fayard, 1992, p. 308.

20. Annales du pèlerinage, $1^{\mathrm{er}}$ août $1923, \mathrm{n}^{\circ} 6, \mathrm{p} .8$. 
vaient encore défendre ce projet extérieur et lancer la propagande alors captée par l'ossuaire de Douaumont ( $\mathrm{M}^{\mathrm{gr}}$ Ginisty le présenta précisément à Vannes en novembre 1923). L'avancée des travaux, surveillée par les pèlerins et la presse, était en effet primordiale. De l'été 1922 au printemps 1924, la maçonnerie des fondations avait été volontairement menée à un rythme très soutenu pour frapper l'opinion. Le mausolée devait en effet commencer à sortir de terre pour l'été 1924. Les festivités du troisième centenaire peuvent être considérées comme la véritable inauguration du monument. Le 26 juillet, la grand-messe du pardon (en présence, à défaut de Foch, du maréchal Franchet d'Esperey) eut lieu dans la prairie du monument, permettant pour la première fois une appropriation de l'espace mémoriel et du chantier par les pèlerins. Or, la solennité de l'anniversaire, aidée par un soleil radieux, avait amené la foule (45000 pèlerins contre 9000 en 1920 ou 12000 en 1921 pour s'en tenir aux chiffres des organisateurs). À défaut du monument lui-même, $\mathrm{M}^{\mathrm{gr}}$ Gouraud pouvait imposer son esplanade bucolique propice aux rassemblements de masse, dans une démonstration qui traduisait bien ce que Michel Lagrée a dénommé « la tentation multitudinaire ${ }^{21}$ " de l'Église du temps : «Comme le disait $M^{\text {gr }}$ l'Évêque, la foule occupait un espace de 100 mètres sur 160 , soit $16000 \mathrm{~m}^{2}$. À trois personnes en moyenne par $\mathrm{m}^{2}$, la foule de la grand-messe peut être évaluée à 45000 ou 50000 personnes $^{22}$. "

L'intégration spatiale du monument et du pèlerinage, qui fit par la suite l'équilibre du lieu, était d'autant plus importante qu'elle n'avait pas été réussie auparavant. La première cérémonie publique - celle de la bénédiction de la première pierre - en granit de l'île aux Moines - par le nonce Cerretti s'était révélée un échec. Organisée à la fin de l'année des pèlerinages, le dimanche $1^{\mathrm{er}}$ octobre 1922, à défaut du 26 juillet pour cause de vacances du nonce, la journée avait été gâchée par la pluie intense - annonciatrice des problèmes d'humidité postérieurs - qui avait contraint à organiser la cérémonie à la basilique seulement remplie. Profitant d'une courte éclaircie, la bénédiction avait été menée au pas de course, après une procession champêtre et boueuse sans éclat. La légitimation de cette cérémonie que la hiérarchie catholique donna par la suite ne tenait donc pas : "Les évêques de Bretagne avaient décidé qu'il ne serait fait aucune invitation officielle à cette cérémonie. Il fallait que cette glorification collective de nos morts fût spontanée et populaire ${ }^{23}$. " $M^{\text {gr }}$ Cerretti, lui-même qui avait construit le début de sa nonciature, après la canonisation de Jeanne d'Arc en présence de 80 parlementaires à Rome, en donnant des signes de connivence à la République sur son terrain de la commémoration (visite à l'Arc de triomphe le 2 novembre 1921) ne dut guère apprécier la tournure de ce déplacement provincial froidement décrit par le préfet comme " privé et religieux ${ }^{24}$ ".

21. Religion et cultures..., op. cit., p. 192.

22. Semaine religieuse du diocèse de Vannes, 2 août 1924, n 31, p. 490.

23. Ibidem, 7 octobre $1922, \mathrm{n}^{\circ} 40$, p. 638.

24. Arch. dép. du Morbihan, 2 W 15816 : lettre au ministre de l'Intérieur du 29 septembre 1922. 


\section{La mutation dynamique (1925-1926)}

En 1925, l'Église bretonne gardait le même esprit défensif mais avec des capacités de mobilisation autrement imposantes. Les organisateurs du pèlerinage tinrent une nouvelle fois à lier la grand-messe du 26 juillet au monument. À défaut de la prairie encombrée par $600 \mathrm{~m}^{3}$ de pierres de taille, le parc contigu du petit séminaire fut en effet choisi de manière inédite, au détriment du traditionnel champ de l'épine devant la Scala Sancta. Ce déplacement avait été annoncé, la veille, par la première procession nocturne en direction du monument, modifiant et amplifiant les trajectoires jusqu'alors centrées sur la basilique et le cloître. On peut penser que l'appropriation populaire du monument s'est réalisée alors avec le véritable lancement de la campagne de financement de l'inscription des pierres pour les disparus. Ce démarrage n'a rien de spontané. Il a été construit par la nouvelle géographie mentale du sanctuaire décrite conduisant le pèlerin à ajouter lui aussi sa pierre à celles qu'il voyait sur l'esplanade, et surtout forcé par des techniques de propagande bien affûtées dans les paroisses. Celles-ci furent mises en place en novembre 1925. Cette temporalité du souvenir connut une campagne de presse marquée par un nouvel appel collectif de l'épiscopat régional - le troisième -, avec annonce d'une quête régionale (sauf dans le diocèse de Nantes) et surtout par les articles de Georges de Lys (colonel Georges Fontaine de Bonnerive) dans La Croix, relayée par ses feuilles hebdomadaires de province et des titres bretons (Nouvelliste, Feiz ha Breiz...), ainsi que de Charles Le Goffic dans Le Gaulois du 16 novembre. Ce dernier avait été sollicité par le nouvel évêque de SaintBrieuc $M^{\mathrm{gr}}$ Serrand, un ancien combattant plus favorable au monument que son prédécesseur. Ces textes littéraires, dont on peut d'ailleurs remarquer le caractère assez tardif, contribuèrent à mieux greffer sur le monument à la fois l'épopée bretonne de la Grande Guerre et son corollaire de la victimisation exemplaire régionale. Ils ont donc amélioré la visibilité et la singularité du projet de Sainte-Anne, en particulier en direction des Bretons de Paris.

La propagande, qui était bien le terme employé à l'époque, correspondit à la combinaison de deux savoir-faire, celui des missionnaires diocésains de Sainte-Anne et celui de la Fédération nationale catholique et de son satellite dans le clergé, la PAC ([Association des] Prêtres anciens combattants) impliquée à partir de son congrès régional du 29 juillet 1926 . La campagne de financement du monument aux Morts bénéficia surtout de l'onde de choc dynamique de la structuration militante masculine combattante qui venait de peser - avec succès - sur le Cartel des gauches. Le parallèle peut être fait dans le diocèse de Vannes entre la préparation de la manifestation du 29 mars 1925 et les efforts de l'automne et de l'hiver pour le monument. C'est ainsi qu'en vue de l'inscription des morts - désormais fixée à 200 francs - 10000 circulaires en plus des affiches pour les églises et des tracts ont été diffusées aux paroisses du Morbihan par le relais des délégués cantonaux des prêtres et instituteurs anciens combat- 
tants. Le changement d'échelle de la mobilisation était incarné par un ancien combattant, l'abbé Le Moigno, directeur des pèlerinages, qui se consacra entièrement à partir du mois d'octobre à la prédication en faveur du monument dans les paroisses. Les résultats ne se firent pas attendre. En juillet 1926, la visite de 51 paroisses et l'affichage généralisé a amené 54000 francs de quête et 1000 demandes d'inscription (elles n'étaient que 37 avant octobre). Le succès de la méthode se mesure à la lecture des résultats des inscriptions des autres diocèses, où le seul appel collectif des évêques avait été nettement moins productif : Quimper (76), Saint-Brieuc (73), Rennes (39), Nantes (18).

L'intensification de la mobilisation se révèle par d'autres signes. L'ouverture de deux nouveaux comptes-chèques postaux, en plus des deux existants, pour recevoir les dons en est un. La Semaine religieuse commença à publier - et cela continua jusqu'en 1932 - un tableau des paroisses, avec le nombre de leurs pierres, en n'hésitant pas à adopter le ton du commentaire sportif pour favoriser l'émulation des curés. En juillet 1926, les Annales du pèlerinage - paraissant tous les 2 mois - qui faisaient de même transmirent la publication des noms devenus trop nombreux aux quotidiens régionaux. Le retour de la pratique de guerre des "monuments de papier $^{25}$ " était accompagné de l'appel à des « marraines " finançant les inscriptions des pauvres combattants (une Parisienne d'origine bretonne s'illustra en offrant 4000 francs, soit 20 pierres pour les marins), puis à partir de 1928 de la mobilisation infantile (particulièrement forte à Pontivy) à la manière des croisades passées et en utilisant les mêmes ressorts de sensibilisation ${ }^{26}$. La propagande en elle-même, telle qu'elle était présentée aux prêtres ou directement à l'opinion par les missionnaires de Sainte-Anne, pouvait se révéler oppressante, en particulier à l'égard des femmes. Était souvent soulignée en effet la modicité de l'achat d'une inscription en comparaison de la somme versée par l'État en pensions ou en rentes : "Les veuves ont peut-être des enfants à élever [...] mais ce sont les morts qui les aident à payer les frais de cette éducation ${ }^{27}$ ! " Les prêtres qui négligeaient de mobiliser leurs paroissiens pour le financement du mémorial en préférant ne pas les distraire de celui des œuvres paroissiales étaient tout aussi nettement rappelés à l'ordre par les propagandistes. Enfin, l'euphorie de la victoire contre le Cartel des gauches n'avait pas infléchi le caractère intégraliste du discours autour de Sainte-Anne, bien au contraire puisque la concurrence républicaine municipale continuait de freiner le dynamisme du projet :

25. Marie-Thérèse CLOÎTRE, "Mort, bons morts et bonne mort d'après les " monuments de papier " de la guerre 1914-1918 dans la presse finistérienne ", Des vivants et des morts, Brest, ARS et CRBC, 2004, p. 337-347.

26. "Un enfant de Compiègne, Michel Faure, s'est constitué l'apôtre de nos morts. Malade, il profite d'une petite amélioration pour se mettre en campagne ", Annales du pèlerinage, $1^{\mathrm{er}}$ septembre $1927, \mathrm{n}^{\circ} 18, \mathrm{p} .4$.

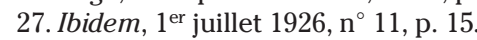


" Il y a, me dira-t-on, dans chaque commune un monument où sont inscrits les noms de tous les morts. Oui, c'est vrai, mais un monument presque païen, où souvent ne figure même pas une vraie croix; et s'il y a une croix, c'est rarement avec la figure du Christ. Qui songera un jour, qui songe déjà aujourd'hui en passant devant ces monuments profanes à réciter une prière pour nos morts? Tandis qu'à Sainte Anne c'est un monument éminemment religieux où les prières pour nos morts dureront autant que le monument lui-même ${ }^{28}$."

La thématique du "réveil catholique ${ }^{29}$ " qui a pu être associée à la période se constate pleinement avec un foisonnement d'idées pour améliorer le financement de la construction du monument : constitution d'un musée breton (poupées et costumes) dans le sanctuaire dont la création est liée au développement d'un tourisme mémoriel, concerts et séances cinématographiques de soutien, appel à la documentation pour un Livre d'or des catholiques bretons morts au front ${ }^{30}$. Mais c'est plus encore la vitalité démonstratrice du sanctuaire qui justifie l'expression à partir de 1926 permettant à l'Église, en particulier par la voix de Buléon, de souligner que la foule rassemblée n'avait rien à voir avec les quelques touristes qu'auraient attirés les premiers sites évoqués ${ }^{31}$. Les fêtes du Bleun-Brug de Sainte-Anne-d'Auray le 16 septembre qui s'intéressèrent beaucoup au Monument est la preuve indirecte de son nouveau rayonnement. Le visage du lieu en était modifié avec l'introduction dans le calendrier du pèlerinage d'une " journée du souvenir ", le $1^{\mathrm{er}}$ dimanche d'octobre. Celle-ci était née en 1926 de la déception de ne pouvoir inaugurer la crypte, lors du pardon de juillet. Elle donna lieu à une cérémonie combattante où l'Église mit en avant, en lui réservant les places d'honneur sur une estrade construite devant le chantier, la présence massive des officiers (le commandant de Bonnerive et le général de Langle de Cary, un des vainqueurs de la Marne), ainsi que les " locaux " qui ont organisé la manifestation vannetaise de la FNC (colonel Fonssagrives en premier lieu, mais aussi le général d'Aboville et le commandant Vannier). Cette mise en scène combattante fut encore marquée l'année suivante lors de l'inauguration et la bénédiction de la crypte - vide -, le 26 juillet, marquée par la célébration matinale du P. capucin Yvon, dont la " poitrine [était] constellée de croix ", le défilé clinquant de presque 500 prêtres et d'une garde d'honneur des évêques réunissant 17 drapeaux d'associations. L'uniforme était de mise puisque les scouts faisaient également leur apparition dans l'encadrement du cortège.

28. Ibid.

29. Voir David BEnSOUSSAN, "Le réveil des catholiques bretons (1924-1926) ", Vingtième Siècle, 57, janvier 1998, p. 57-75.

30. Ce projet n'a pas abouti. Une fois rassemblée, la documentation devait être confiée à un écrivain catholique pour être éditée. Elle a seulement alimenté les Annales du pèlerinage.

31. Voir entre autres les Annales du pèlerinage, $1^{\mathrm{er}}$ septembre 1927, n $18, \mathrm{p} .12$. 


\section{Le temps de la consolidation (1927-1931)}

La survalorisation combattante et masculine - qui se comprend mieux lorsqu'on sait combien l'Église devait faire face, depuis la guerre et les habitudes prises au front, à une importante hémorragie de la pratique religieuse des hommes dans les paroisses ${ }^{32}$ - eut des effets d'exclusion. C'est ainsi que le grand congrès de l'Union catholique diocésaine du 16 octobre 1927 , réunissant 45000 « manifestants-pèlerins » en majorité anciens combattants fut convoqué à Sainte-Anne-d'Auray avec ce mot d'ordre qui rompait avec l'identité habituelle du pèlerinage : "Ce jour-là les impotents seuls avec les femmes et les enfants resteront au foyer ${ }^{33}$. " De fait, à la vue du cortège portant insigne FNC, scindé en 2 colonnes, " en rang par 4 ", et réglé pendant une heure par le colonel Fonssagrives, $\mathrm{M}^{\mathrm{gr}}$ Gouraud, dont c'était le dernier grand discours public, d'ailleurs relayé par haut-parleurs, reconnut que le sanctuaire n'avait jamais connu un défilé aussi martial...

L'année 1927 voit l'extension de la dynamique de propagande en dehors du Morbihan. Après avoir visité une centaine de paroisses dans son diocèse, l'abbé Le Moigno, bien accueilli par Duparc, étend alors ses prédications au Finistère, pendant que $\mathrm{M}^{\text {gr }}$ Picaud, évêque auxiliaire de Vannes, fait de même - quoique bien plus modestement - à Rennes. À Paris, la "Paroisse bretonne " s'investit également en accueillant Le Moigno lors d'une soirée mondaine en janvier, puis en mai lors de la Saint Yves à NotreDame des champs. Intéressons-nous au premier rendez-vous qui rassemble plusieurs personnalités invitées par les jésuites Camenen et Questel : les académiciens Robert et Bordeaux et celui qui le devint en 1930 Le Goffic, les prélats «bretons " Le Hunsec (supérieur général des spiritains) et André du Bois de La Villerabel (archevêque de Rouen), les amiraux Schwerer et Guépratte, le commandant de l'institution des Invalides... Tous réunis pour écouter le concert de la violoniste Corrie Siohan, petite fille de Renan et sœur de Psichari, ils entendirent également l'archevêque de Rouen, traduire, non sans justesse, la fonction centrale du monument dans le travail de deuil collectif pour une région aussi périphérique que la Bretagne : « Le Monument de Sainte-Anne était nécessaire [...] parce qu'il y a des morts qui n'ont pas de tombeaux, parce que les cimetières du front sont trop lointains pour beaucoup, et parce que ceux qui ont des tombes sur la terre natale voient leurs efforts dispersés. Il faut que nous puissions faire la synthèse de tous nos sentiments et de tous nos souvenirs ${ }^{34}$."

Sur place, la crypte du monument dédiée à Jeanne d'Arc dont l'ornementation était alors réduite à une statue de sainte Anne, un autel provisoire de bois - rappelant l'austérité des célébrations du front - et à deux tombes symboliques sans corps ${ }^{35}$ mais avec deux croix de bois portant un

32. Michel LAGRÉE, Religion et cultures... op. cit., p. 79.

33. Annales du pèlerinage, $1^{\mathrm{er}}$ septembre $1927, \mathrm{n}^{\circ} 18, \mathrm{p} .6$.

34. Ibidem, $1^{\mathrm{er}}$ mars $1927, \mathrm{n}^{\circ} 15, \mathrm{p} .7$.

35. Dont " les cadavres gisent peut-être dans les tranchées inconnues [ou] dans les abîmes de l'océan " : extrait de la lettre collective des évêques bretons, Ibid., 2 avril 1932, n 14, p. 220. 
casque de poilu et un béret de marin, accueillit à partir de novembre 1926 des messes matinales quasi quotidiennes à l'intention des soldats et devient lieu d'indulgences. La crypte, " éclairée par d'étroites verrières d'un ton jaune " et souvent appelée la " chapelle du Souvenir breton ", reçut dès 1928 la visite annuelle d'une centaine de pèlerinages paroissiaux. Les pèlerins qui portaient - et ce de manière nouvelle comme à Lourdes - des insignes où figurait le monument, ponctuaient là leur parcours dans le sanctuaire par un Libera chanté. En 1929, on commença également à y célébrer la Toussaint et à commémorer le 11 novembre.

Ce quotidien de messes et de prières convainc que le purgatoire n'était pas à son crépuscule à Sainte-Anne-d'Auray. Dès 1922, pour relayer les messes attendues de la hiérarchie au moins jusqu'en 1942, les chanoines Buléon et Le Garrec, très attachés à lier le sanctuaire au dialogue avec les trépassés, avaient en effet prévenu que le monument devait être à la fois " un arc triomphal " et " une chapelle funéraire " puisque les morts bretons de la Grande guerre, « martyrs de la Grande Guerre ", n’étaient " pas nécessairement des saints ${ }^{36}$ ». En suivant le travail national de Guillaume Cuchet $^{37}$, on se rend compte cependant que l'épiscopat breton, tout en restant tributaire de cette dévotion bien enracinée dans ce cadre régional, nourrissait quelques scrupules à faire aussi automatiquement de ces martyrs sublimés de la guerre des âmes du purgatoire.

Le chantier tournait à plein durant ces années, marqué par les échafaudages et donc l'élévation progressive du monument : les huit chapiteaux de soutien de la voûte où furent gravées les années de guerre (1927), l'étage composé par la galerie extérieure et la lanterne $(1928,1929)$, les deux escaliers d'accès et la coupole haute de 45 mètres (1930-1931). Les dépenses augmentèrent tout autant, passant de 400000 francs annuels à 600000 francs en 1930 (atteignant alors un total de plus de 4 millions de francs). La campagne de propagande sur les inscriptions, source principale de financement, ne devait pas donc pas faiblir. De 1927 à novembre 1928, une cinquantaine de nouvelles paroisses ont été visitées dans le Morbihan par les propagandistes du monument, une centaine dans le Finistère, 70 en Ille-et-Vilaine et une dizaine dans les Côtes-du-Nord, département qui concentra ensuite l'attention avec plus de 100 paroisses visitées jusqu'en 1932. La répétition des appels aux souscripteurs fortunés atteste les besoins élevés de l'Église. Ceux-ci se manifestèrent dans les dons pour le mobilier et la décoration de la crypte (un prêtre et sa sœur financèrent à eux seuls l'autel du diocèse de Vannes en offrant 10000 francs) qui mobilisa tout autant religieuses et dames de l'œuvre des tabernacles. Ils se concentrèrent également sur la belle et lourde croix en bronze de la coupole rappelant un calvaire breton, bénite et montrée à dessein de financement lors du pardon du 26 juillet 1930. Le monument prit sa forme extérieure définitive grâce aux travaux de finition de l'année 1931 (pose des

36. Sainte Anne et le monument des Bretons morts à la guerre, Vannes, Lafolye, 1922, p. 1-2.

37. Le crépuscule du purgatoire, Paris, A. Colin, 2005. 
ardoises de Sizun sur une flèche incurvée et de la croix à 52 mètres). Le travail porta également sur le remblais de l'esplanade mettant à contribution, à partir de mars 1931, les bonnes volontés locales et les séminaristes. La maison qui bouchait encore la visibilité du monument fut rasée, ce qui permettait désormais aux pèlerins arrivant de la gare de Pluneret d'apercevoir, dès leur arrivée dans le bourg et ce à la faveur d'une longue ligne droite, le site mémoriel.

\section{La grande journée du 24 juillet 1932}

Durant de longs mois, toute la tension du sanctuaire se concentra sur la réussite de la journée d'inauguration du monument central fixée au dimanche 24 juillet 1932, soit deux semaines avant celle de l'ossuaire de Douaumont. Rien ne fut laissé au hasard d'une préparation d'autant plus maîtrisée qu'elle a été précoce, mobilisatrice et inventive. On en jugera par la composition festive du site : pavois tricolore de 7 mètres sur 4 offert par l'île de Groix, immense arc de triomphe pour la rue de la gare confectionné par 4 prêtres représentant le calvaire de Saint-Thégonnec et réaffirmant en breton et en français la prophétie de Calloc'h, reproduction cartonnée des stations du chemin de croix par plusieurs congrégations religieuses, peinture de grands tableaux retraçant l'histoire de la Bretagne par le petit séminaire de Sainte-Anne-d'Auray pour les rues d'accès au sanctuaire, pose de guirlandes dans le bourg par une vingtaine de séminaristes de Vannes et les habitants, coupe de 400 sapins chez deux grands propriétaires de Landévant pour servir notamment de mâts aux drapeaux...

Ce " décor de cinéma " attendait ses figurants. Il convient d'insister ici sur l'habileté de l'Église pour ouvrir au maximum le rassemblement aux anciens combattants. La préparation puise ici ses racines dans les cérémonies passées évoquées. Mais il faut reconnaître encore des bonifications. L'idée maîtresse a été d'impliquer les régiments et les corps d'armée bretons en réclamant leurs drapeaux pour la cérémonie. Ce projet, né dans l'esprit du comité d'exécution durant l'été 1930 pour des raisons de financement que l'on exposera par la suite, a été particulièrement entendu des associations. L'émulation joua en effet à plein puisque l'Union nationale des combattants - conservatrice - fournit 500 drapeaux, tandis que l'Union fédérale - de centre gauche - en donna 200 après avoir elle aussi appelé ses adhérents au rassemblement dans la presse. La participation de cette dernière association que L'Ouest-Éclair expliqua autant par sa volonté de " satisfaire ses membres croyants que pour apporter son hommage ${ }^{38}$ " revêt une grande importance dans l'histoire du monument. Elle signifie que, contrairement au début des années 1920, celui-ci est désormais perçu non seulement comme un espace consensuel d'Union sacrée, mais bien comme le seul lieu majeur d'identification régionale de la commémoration de la grande guerre.

38. Article de Léon Le Berre, numéro du lundi 25 juillet 1932. 
Si le glissement perçu correspond aux phases d'appropriation du monument décrites entre la première bénédiction de Cerretti et la participation de son successeur Maglione à la journée du 24, on ne manquera pas d'insister sur la conjoncture du début des années 1930. Celle-ci voit un glissement fondamental de la hiérarchie catholique ${ }^{39}$ - que personnifie le nouvel évêque de Vannes $\mathrm{M}^{\mathrm{gr}}$ Tréhiou par rapport à son prédécesseur ou à l'échelle nationale le cardinal Liénart -, vers l'affirmation d'un pacifisme favorable à Briand qui a pour effet de " recentrer " l'Église et d'augmenter son crédit dans la société du temps. Le monument devient ainsi à partir de 1931 et pour la première fois dédié à la paix, introduisant une inflexion par rapport à la seule invocation de Jeanne d'Arc des années 1920. L'équilibre favorable - un gouvernement de centre-gauche pour un épiscopat qui n'était plus lié à l'Action française - se manifesta à la tribune d'honneur érigée près du monument. Certes, le préfet en déplacement à Paris n'était pas présent - ce qui n'a pas échappé à L'Ouest-Éclair se demandant si les morts de Bretagne ne valaient pas au moins une présence préfectorale et davantage de sénateurs " embusqués " que les deux parlementaires (Grand et Garnier ${ }^{40}$ ) -, mais l'État était notablement représenté par le chef de cabinet du préfet Benedetti, ainsi que par le sous-préfet de Redon. $\mathrm{M}^{\mathrm{gr}}$ Tréhiou, bien plus ouvert qu'un $\mathrm{M}^{\mathrm{gr}}$ Ginisty - évêque de Pie X - au même moment à Verdun, salua avec insistance cette présence qui rompait avec les pratiques antérieures et que vint d'ailleurs entretenir dans la foulée une Légion d'honneur accordée au chanoine Quelven. La dizaine d'officiers et d'amiraux, la centaine d'officiers d'active, les deux drapeaux des fusiliers marins et du $62^{\mathrm{e}} \mathrm{RI}$ escortés par des pelotons encadrant les orateurs à la tribune complétaient cet investissement officiel, entraînant d'ailleurs des pèlerinages spontanés de soldats vers Sainte-Anne (73 fantassins du 71 ${ }^{\mathrm{e}} \mathrm{RI}$ de Saint-Brieuc).

L'afflux de participants (125000, pour trancher entre des estimations allant de 100000 à 150000 personnes), aux deux tiers masculins, a donc fait de cette journée le plus grand rassemblement breton de l'entre-deuxguerres, ce qui a fortement mobilisé les journaux régionaux et, dans un style plus sobre quoique sans euphémisation, plusieurs titres parisiens Le Figaro, L'Écho de Paris, Le Journal, La Croix. 7000 autos réunies, non sans ralentissements, dans quatre parkings ${ }^{41}, 25000$ voyageurs en train acheminés par 14 convois supplémentaires dont un de Paris, quatre lieux de rassemblement réservés aux associations d'anciens combattants pour leur repas indiqués par un plan édité par L'Ouest-Éclair qui sort pour l'occasion un numéro spécial vendu 10 centimes $^{42}, 140$ secouristes, 400 scouts enca-

39. Frédéric LE MoIGNE, Les Évêques français de Verdun à Vatican II. Une génération en mal d'hérö̈sme, Rennes, Presses Universitaires de Rennes, 2005, p. 51-64.

40. À l'inverse, il y avait 7 députés : Guillois, Pézet, Desgranges, de Juigné, Le CourGrandmaison, Le Pévédic, Barbot.

41. Une route d'accès réservée avait cependant été prévue pour les mutilés. Le chiffre des camions à bancs, principal moyen d'acheminement depuis les années 1920, n'est malheureusement pas connu.

42. La modernité de sa maquette doit être de nouveau notée avec une couverture 
drant la foule en compagnie de nombreux commissaires, 1000 couverts servis pour le clergé sous le préau du petit séminaire, voici quelques éléments de la logistique d'un rassemblement devant beaucoup à la discipline combattante (qui aurait fait fuir les pickpockets et les camelots pourtant attendus). Le savoir-faire engrangé en 1925 se révèle une nouvelle fois payant avec d'ailleurs le même maître de cérémonie, le colonel Fonssagrives, relayant l'interdiction par le clergé de toute consommation d'alcool fort et canalisant les hommes par unités dans des carrés diocésains délimités à l'avance. Cette totalisation masculine eut logiquement des conséquences d'exclusion des femmes. Une seule d'entre elles (madame Monphous, veuve d'un colonel et infirmière mutilée) fut invitée à l'estrade officielle. Surtout, les femmes se virent, l'après-midi, interdire l'accès à l'esplanade saturée. Pour autant, il faut se garder d'effacer la dimension familiale du rassemblement. Un journaliste parisien, humant l'exotisme du lieu ou peut-être inspiré par la vue de quelques soldats malgaches dans l'assistance, évoque un dimanche d'affluence à l'exposition coloniale contemporaine. Les journaux firent leur chou gras des photographies de piquenique, surtout quand elles fixaient pour les feuilles parisiennes quelques coiffes, chapeaux ronds et sabots. La journée tient également du pèlerinage sous bien des aspects, ne serait-ce que parce qu'elle s'insère dans le calendrier de la sainte Anne sans d'ailleurs gêner l'assistance du 26 (40000 pèlerins). C'est la synthèse de ces deux identités - le meeting moderne et le pardon breton (" les gilets de velours, les vestons, ou les vestes d'uniformes ${ }^{43}$ ") - qui lui a donné sa couleur et sa fréquentation.

Le récit de cette journée au temps couvert et frais (mais qui, grâce aux prières récitées pendant de longues semaines en amont, n'a connu que de courtes averses peu gênantes) s'arrêtera sur la messe pontificale du matin pour la première fois célébrée depuis la loggia - Notre-Dame de la paix décorée de longues tentures blanches semées d'hermines, avec son nouvel autel en granit rose de Ploumanac'h, financé par les Croisés de l'Eucharistie du Morbihan, " sobre comme un dolmen ", installé difficilement (il pesait $2000 \mathrm{~kg}$ ) deux jours auparavant. Le déploiement des célébrants (une vingtaine de prélats dont le nonce) était cependant rendu difficile dans cet espace surélevé du chœur ne dépassant pas $150 \mathrm{~m}^{2}$. Cela n'a pas nui à la solennité liturgique, favorisée par une chorale de 800 exécutants dirigée par le chanoine Pirio, maître de chapelle de la cathédrale de Vannes, et par la réplique des fidèles en particulier pour l'envoi que le rédacteur diocésain trouva " autrement impressionnant " que le chant de clôture du congrès eucharistique de Dublin du 26 juin ${ }^{44}$. L'après-midi commença par le défilé des drapeaux des unités d'anciens combattants - dont celui de la PAC nationale porté par le chanoine Umbricht et celui des fusi-

originale comportant un portrait de l'entrepreneur Huchet assis sur un bloc de granit et une page entièrement photographique (9 portraits d'évêques et du nonce).

43. L'Éclair du Finistère, dimanche 31 juillet 1932.

44. Semaine religieuse du diocèse de Vannes, 30 juillet 1932, n 31, p. 497. 
liers par le chanoine Le Helloco - du terrain de la Scala Sancta vers les escaliers du monument, arrachant, à sa conclusion, les applaudissements de la foule, tandis que commençait à retentir la cantate triomphale " aux Morts de Bretagne " spécialement composée pour l'occasion par le Chanoine Le Dorze, curé de Saint-Patern de Vannes et l'organiste rennais CharlesAugustin Collin ${ }^{45}$, et qui fut par la suite éditée.

La mémoire collective a retenu le discours de $\mathrm{M}^{\mathrm{gr}}$ Duparc, " cette figure de vitrail creusé par l'apostolat " comme le qualifie de manière inspirée $L e$ Cri du poilu d'août 1932. Prononcé à l'issue de la grand-messe du matin, ce bel exemple d'éloquence catholique polie par l'expérience (le prélat a alors 75 ans) et que rendent particulier dans le discours hiérarchique de l'époque le mysticisme bas-breton et le lyrisme suranné - diffusé par les moyens modernes du micro - doit être lu en corrélation avec le discours prononcé le 7 août suivant à Vannes, lors de la commémoration du $4^{\mathrm{e}}$ centenaire de l'union de la Bretagne à la France. Du lien entre la grande patrie - d'ailleurs égratignée pour " le retour à ses vieilles erreurs ${ }^{46}$ " d'avant l'Union sacrée et la petite patrie, on ne peut retenir ici que le rôle assigné aux morts de la Grande guerre. Duparc affine en effet la représentation du monument :

"Sur une sépulture des catacombes, j'ai lu cette inscription : que chacun des frères qui aura lu ces noms prie Dieu. [...] Si le nom de tous les Bretons de la guerre était gravé sur le mur d'enceinte qui encadrera ce monument, il faudrait étendre le mur aux proportions du village tout entier, devenu un vaste mémorial funèbre. [Nos morts] représentent à eux seuls la population d'une grande ville ${ }^{47}$."

Peut-on mieux symboliser le bastion catholique qu'est le monument de Sainte-Anne-d'Auray, bien installé dans sa ruralité (dont la population dépasse la grande ville à l'heure de l'émigration bretonne...), à la jonction de l'Armor et de l'Argoat et faisant ainsi l'équilibre entre le souvenir des marins ${ }^{48}$ et des paysans, et dans sa fécondité multitudinaire sacrificielle? Le reflet des morts sur l'assistance du 24 juillet à Sainte-Anne-d'Auray a servi au triomphalisme de l'Église. C'est donc bien ici qu'il faut évoquer la fameuse inflation des 240 voire des 250000 morts dont le monument de Sainte-Anne-d'Auray a été le principal vecteur dans la société bretonne. $\mathrm{M}^{\mathrm{gr}}$ Serrand, l'autre orateur de l'après-midi avec son discours ancien combattant ${ }^{49}$, nous y invite, lui qui reprit en touts points les conclusions de Le Goffic et de Bonnerive sur le "sixième des morts français" :

45. Georges Provost, La Vie musicale dans le diocèse de Rennes au XX APO 35-AHID, 2005, p. 66-68.

46. Art. cité, Semaine religieuse du diocèse de Vannes, p. 500

47. Ibidem, p. 499.

48. Le monument national de la pointe Saint-Mathieu, ciselé par Quillivic et inauguré en 1927, était dédié aux seuls marins. Sainte-Anne d'Auray a toujours été liée au souvenir des disparus en mer, comme le prouve aujourd'hui un touchant mémorial près du monument de la Grande Guerre.

49. Chaleureusement applaudi par la foule à la fin de son discours, l'évêque de SaintBrieuc pouvait cependant être déçu. Les haut-parleurs étaient rapidement tombés en 
«Vous connaissez tous le chiffre de nos morts : $240000 \ldots$ Vous êtes vous rendu compte à quel point il est élevé?... La Bretagne a [...] une population de 3 millions, c'est-à-dire à peu près la treizième partie de la population de la France qui est de 39 à 40 millions. Voulez-vous multiplier 240000 par 13?... À quel chiffre arrivez-vous?... Trois millions cent vingt mille exactement. C'est-à-dire un peu plus de deux fois le chiffre total des morts [...]. La conclusion?... La proportion des morts en Bretagne est au moins 2 fois plus forte que la proportion des morts dans les autres régions françaises prises dans leur ensemble. N'avons-nous pas le droit de nous replier douloureusement sur nous-mêmes après cela et de pleurer à part, et de commémorer à part, par un monument à nous ${ }^{50}$ ?..."

La démonstration est symptomatique de cette ivresse multitudinaire que goûtait alors l'orateur devant plus de 100000 auditeurs acquis à sa cause, et qui lui fit conclure ainsi : «Ici, mieux qu'à Douaumont, mieux qu'à NotreDame de Lorette, mieux qu'à Dormans, mieux même qu'à l'Arc de Triomphe, la flamme sera ravivée... Ici s'est allumé un foyer où les âmes des Bretons se réchaufferont sans arrêt les unes contre les autres dans l'amour de la grande et de la petite Patrie ${ }^{51}$. " De même que c'est en 1925, lorsque l'Église bretonne, après avoir jusqu'alors évoqué le chiffre de 200000 disparus de la grande guerre, ajoute 50000 unités pour mieux les associer aux 250000 manifestants régionaux contre le Cartel ${ }^{52}$, de même c'est le 24 juillet 1932 qu'elle ajoute aux 240000 morts de son monument les 150000 participants pour achever sa représentation de masse. L'historien, tout en souhaitant que le diocèse de Vannes et les associations d'anciens combattants (Souvenir français et UNC) qui entretiennent actuellement le monument puissent corriger les deux grands panneaux du mémorial indiquant encore l'hommage aux 240000 morts bretons de la Grande Guerre, n'est cependant pas étonné de la pérennité du nombre tant sa symbolique est associée - plus encore qu'à la mémoire - à la légitimation du lieu.

\section{L'achèvement du mémorial (1933-1937)}

On peut cependant avec un peu d'effort échapper au sortilège ${ }^{53}$ de ce dénombrement en se rendant au mémorial lui-même et en comptabilisant

panne (pareille mésaventure arriva en juillet 1940 à $\mathrm{M}^{\mathrm{gr}}$ Roques pour sa première allocution à Sainte-Anne), et ses phrases prononcées de la loggia tombèrent « au ras du monument ". Les comptes-rendus journalistiques (L'Écho de Paris parle cependant de " $\mathrm{M}^{\mathrm{gr}}$ Ferrant ") firent de leur mieux pour redonner son importance au discours, surtout les Semaines religieuses qui le publièrent en entier.

50. Semaine religieuse du diocèse de Vannes, 6 août 1932, n 32, p. 512-513.

51. Ibidem, p. 514

52. Voir le discours de $\mathrm{M}^{\mathrm{gr}}$ Gouraud, idem, 4 avril 1925, $\mathrm{n}^{\circ}$ 14, p. 219.

53. Celui-ci a joué sur bien des plumes. Arrêtons-nous seulement sur celle d'Éric MuRAISE (colonel Suire) qui, malgré son appartenance au SHAT et la co-écriture d'une Histoire de la Première Guerre mondiale pour Fayard, relaya sans problème le chiffre des " 240000 " dans Sainte Anne et la Bretagne, Paris, Fernand-Lanore, 1980. L'archéologie de ce chiffrage né dès la guerre et qui est loin d'être l'unique décompte fantaisiste du temps, la diffusion régionaliste, puis sa tardive contestation, est un sujet en lui-même. 
les chiffres des disparus inscrits sur les plaques paroissiales du mur d'enceinte en 1934 et 1935. Celles-ci reproduisent en effet, grâce au sérieux du collectage effectué pour toucher le maximum de familles, les chiffres des monuments aux morts et des mairies devant aboutir à la fourchette des 110140000 disparus reconnus par l'historiographie. Le mur d'enceinte de 450 mètres de long a été édifié pour la majeure partie en 1933 par 30 maçons et tailleurs de pierre. L'inscription des noms, qui était fortement attendue des premiers souscripteurs, a commencé en février 1934, donnant cette identité d'immense table mémorial au site. Comme une extension de l'aménagement des églises paroissiales, le mur était ponctué par les 14 stations du chemin de croix de l'artiste des Seiz Breur de Sarzeau au style dépouillé, Xavier de Langlais. Sa production avait été un argument de propagande, comme le prouve la mise en avant de sa XI ${ }^{\mathrm{e}}$ station - unique élément du mur sculpté par le Lyonnais Jacques Ballanche - lors de la journée du 24 juillet 1932, ainsi que l'esquisse contemporaine largement diffusée dans la presse associant le sacrifice du combattant à celui du christ. Ce n'est donc pas un hasard si les noms des régiments bretons et des grandes batailles où ils furent engagés entouraient les stations du chemin de croix, tel que l'avait promis $\mathrm{M}^{\mathrm{gr}}$ Tréhiou dans son appel aux anciens combattants de 1932, en leur attribuant d'ailleurs l'initiative du projet (à tort puisqu'il s'agit, comme on l'a vu, du comité d'exécution) pour mieux les motiver.

Le 24 juillet, qui doit être également considéré comme la grande journée de financement du monument, fait en effet césure. Après cette date, la propagande fut un peu délaissée dans les feuilles catholiques et dans les diocèses, comme le prouve le départ en paroisse de Le Moigno (à Guiscriff, ce qui en fit pour l'Emsav catholique un nouveau Perrot oubliant son titre de chanoine donné en compensation), remplacé par l'abbé Le Callet plus discret, puisque l'Église considérait que, grâce à la nouvelle image du monument façonnée par le succès du 24 juillet relayé par la presse, celui-ci avait moins besoin d'être défendu. Cela s'est révélé payant puisque les demandes d'inscription n'ont pas cessé ajoutant 4000 noms aux 4500 collectés avant 1932. Cette campagne d'inscriptions a-t-elle été pour autant un succès? Si l'on s'en tient aux espoirs formulés discrètement en 1926, à savoir environ 20000 noms (et qui n'ont jamais été plus hauts), il est possible d'en douter. Seul le diocèse de Vannes a donné entière satisfaction, avec seulement 2 paroisses sans nom (Saint-Malo de Beignon et Le Temple), permettant de recouvrir à lui seul la moitié du mur d'enceinte avec une densité de noms bien supérieure à l'autre partie (le contraste est ainsi net derrière le monument central à la jonction avec le diocèse de Nantes). Cette imposante uniformité morbihannaise mériterait assurément d'être mieux disséquée pour distinguer les meilleurs élèves (les îles en particulier qui ont été parmi les premières à souscrire massivement) ${ }^{54}$. Elle a incontestablement débordé sur les marges limitrophes des autres départements. C'est vrai en parti-

54. C'est ainsi que pour l'île aux Moines et Arz, il y eut plus de noms gravés que de morts annoncés dans la paroisse. 
culier de tout le nord-ouest de la Loire-Inférieure dans la sphère d'influence du pèlerinage, du sud des Côtes-du-Nord (24 inscriptions de Loudéac contre une seule de Lannion), et du Finistère sud, même si dans ce dernier cas le contraste est net avec le centre du diocèse et non avec le Léon souscripteur). Cette géographie reste cependant fortement dépendante de la réception particulière d'un recteur ou de son indifférence.

En règle générale et sauf exception des pays très pratiquants, le financement extérieur au Morbihan a été moins populaire que dans le diocèse de Vannes. C'est vrai en particulier des grandes villes (les 44 noms de Rennes comptent ainsi 11 particules). Il n'est d'ailleurs pas étonnant que l'Église, plus respectueuse des hiérarchies que ne l'étaient les monuments aux morts de l'égalitarisme républicain, ait tenu à inscrire les grades sur la pierre; ce qui avait pour effet de bousculer parfois le classement alphabétique (la paroisse Saint-Patern de Vannes inscrit ainsi en première position son héros, le lieutenant Xavier Desgrées du Loû). Les prêtres n'étaient pas séparés de leurs paroissiens (la paroisse de Noyal-Pontivy a ainsi payé pour son vicaire), même si le diocèse de Quimper - le seul à avoir publié un livre d'or en Bretagne - a inscrit 3 noms " à la mémoire des séminaristes et prêtres " et si les principales plaques collectives concernaient le clergé ( " 75 morts du clergé nantais ", frères de Ploërmel, jésuites, capucins, bénédictins, spiritains bretons). Il n'y a pas eu une vraie volonté de graver en priorité les religieux comme il en avait été question en 1930 pour lutter contre la rumeur infâme. L'inscription familiale est bien plus imposante, visible avec les disparus d'une même famille (père et fils, et surtout frères). D'ailleurs, le comité avait accepté des paiements uniques pour ces cas (ou une remise de 100 francs pour deux beaux-frères ou pour un oncle et son neveu mais pas pour des cousins germains), s'ajoutant à l'étalement de paiements acceptés pour tous. Enfin, on notera les inscriptions singulières : celle d'une femme de Saint-Nazaire, plusieurs noms des départements français (une dizaine dont quatre de Paris) et de l'étranger (plaque de reconnaissance de la Belgique et du Luxembourg, inscription d'un ressortissant anglais). Malgré ses insuffisances par rapport à l'inscription exhaustive municipale et ses limites régionales, le mur d'enceinte reste impressionnant parce qu'il matérialise sur 450 mètres la mort de masse et qu'il fait ainsi le lien entre le deuil individuel, familial, paroissial, scolaire ('institution notreDame de Guingamp rend ici hommage "à ses 146 morts "), et le deuil fabriqué à Sainte-Anne - celui de 5 diocèses et de la petite patrie bretonne.

En 1932, les frais engagés pour l'édification du monument s'élevaient à plus de 5 millions de francs. Ce fut la somme que reconnut officiellement l'Église ${ }^{55}$, oubliant donc les dépenses du mur d'enceinte, des chapelles de la crypte (1933-1941), de la mosaïque du Monument (1936). L'étirement de ces travaux de finition convainc cependant que la gestion, certes rendue difficile par l'augmentation du coût de la construction par rapport aux pré-

55. Voir Le Pèlerin de Sainte Anne qui se fiait à la comptabilité du 28 mars 1941, date d'achèvement du mémorial (numéro 263 de janvier-mars 1971, p. 28). 
visions de départ, a été maîtrisée. Contrairement à d'autres constructions d'Église - on peut citer ici la coûteuse basilique souterraine de Lourdes dans les années 1950, qui greva le budget du sanctuaire à cause de la gestion de $\mathrm{M}^{\mathrm{gr}}$ Théas " réparée " par la haute hiérarchie -, le mémorial de Sainte-Anned'Auray n'a pas été un gouffre financier. Cette prudence comptable - qui doit être prise en compte dans l'évaluation d'un épiscopat - est incarnée par $\mathrm{M}^{\mathrm{gr}}$ Tréhiou, bien conseillé par l'économe du sanctuaire l'abbé Allanic. À partir de 1934, l'évêque de Vannes réduit ainsi les dépenses du diocèse pour le mémorial au profit de la construction de son grand séminaire, notamment en stoppant les projets initiaux de statue à Jeanne d'Arc (malgré des souscriptions individuelles encore reçues) et de portail monumental (celuici eût d'ailleurs gâché la perspective du monument nécessaire aux grands rassemblements de masse). Il cherche également à faire participer intégralement les autres évêchés au financement des chapelles de leur crypte, ce qu'il réussit avec la plus grande difficulté pour celui de Nantes car il faut attendre 1937 et le remplacement de $\mathrm{M}^{\text {gr }}$ Le Fer de La Motte par $\mathrm{M}^{\text {gr }}$ Villepelet pour que la Loire Inférieure, dont le diocèse n'avait donné jusque-là que 100000 francs, n'organise une seconde quête pour le monument (après celle de $1922 . .$.$) et la campagne de propagande des missionnaires de Sainte-Anne.$

L'architecture de finition du mémorial n'est pas la moins intéressante à analyser en raison des choix de la statuaire. La mosaïque bleue aux rayons d'argent, notamment, aboutit au sommet de la voûte à l'inscription du mot " Pax ». Les chapelles de la crypte concentrent un programme mis en lumière par l'électricité, qui se veut le grand récit de l'épopée catholique bretonne grâce aux statues de saints, aux retables et aux bas-reliefs. La division diocésaine accentue l'intérêt comparatiste, depuis la chapelle d'accès de Vannes jusqu'à celle de Nantes, en passant par celles de Saint-Brieuc, Quimper et Rennes (dans un ordre qui d'ailleurs ne correspond pas à celui du mur d'enceinte extérieur, Vannes, Nantes, Quimper, Saint-Brieuc, Rennes). Les chapelles centrales des trois diocèses sont composées à l'identique autour d'un motif central (scène de la nativité ciselée par Le Bozec pour Saint-Brieuc, remplaçant un projet de crucifixion initial, Sainte Anne la Palud, et Notre Dame de la paix en hommage au cardinal Charost), puis cumulant les statues des saints des diocèses (sculptées par Tardivel pour Rennes, et par Euzen, Donnard et Santelli pour Quimper; avec d'ailleurs un jeu de glissement pour le saint Yves de Le Bozec passant de Saint-Brieuc à la sixième chapelle, qui était collective, pour mieux accompagner saint Michel et marquer ainsi le lien entre le saint patron breton et celui de la France). Les chapelles de Vannes et Nantes se répondent puisque, autour de Jeanne d'Arc pour le premier, de Rogatien et de Donatien pour le second, se trouvent les retables bas-reliefs d'autant plus symétriques qu'ils ont été composés par Jules-Charles Le Bozec. L'artiste de Mellionec, membre des Seiz Breur, était encore davantage mis à l'honneur puisque c'est lui qui cisela les deux grands bas-reliefs de l'extérieur encadrant la porte d'entrée de la crypte, ce qui accentuait l'unité générale avec cette sculpture de la taille directe caractéristique du style régional. 
Si le retable de Nantes en resta à l'évocation des grandes figures du diocèse, celui de Vannes est plus intéressant. Le premier bas-relief évoque certes Beaumanoir et Richemont mais ajoute en son centre $\mathrm{M}^{\mathrm{gr}}$ Gouraud qui, inspiré par un ange, a la vision du monument à construire pour les combattants dont un groupe se dessine derrière lui, portant un mourant. Ce dernier évoque-t-il déjà Jean-Pierre Calloc'h représenté assis écoutant sa Muse? Tandis que le second représente Saint Patern, sous les traits de $M^{\text {gr }}$ Duparc, bénissant la mer et conduisant femmes et hommes en costume traditionnel ainsi que Nicolazic et Charles de Blois. Le peuple se retrouve davantage à l'extérieur sous le bras protecteur des allégories de la Victoire et de la Paix - ce qui atténue d'ailleurs la marque religieuse. Restaient enfin les inscriptions qui ont été décidées personnellement par $\mathrm{M}^{\mathrm{gr}}$ Tréhiou en 1936. Celle de l'entrée de la crypte en latin - «Spes illorum immortalitate plena est " - a été trouvée sans difficulté. Le prélat bretonnant chercha en vain à imposer un vers de Calloc'h, ce qui se révéla impossible du fait des différences de dialectes et des difficultés de traduction, et se résolut à l'inscription simple des monuments aux morts qu'il n'aimait guère : " La Bretagne à ses enfants - Breiz d'he bugale karet. "

L'acmé mobilisatrice du 24 juillet 1932 n'a pas entraîné d'essoufflement cérémoniel durant le reste de la décennie. L'effort a été en particulier diocésain avec des journées marquées par la bénédiction des autels de la crypte, en particulier celle du 22 juillet 1934 pour Rennes présidée par $\mathrm{M}^{\mathrm{gr}}$ Mignen et marquée par le pèlerinage militaire des Chasseurs à pied. Le Pardon de la fin juillet intégra encore davantage le mémorial, notamment lors de la procession nocturne du 25 juillet, lorsque l'autel extérieur, fortement éclairé par 4 projecteurs et ainsi " phosphorescent ${ }^{56}$ ", répondait aux flambeaux des pèlerins. Et si la grand-messe du mémorial déserta le 26 juillet le mémorial pour la Scala Sancta (sauf en 1936), la clôture du pèlerinage par l'évêque de Vannes se faisait immanquablement là.

À l'échelle nationale, l'époque fut marquée par les grands pèlerinages européens d'anciens combattants pour la paix de 1934 et 1936 à Lourdes, fortement encouragés par la nouvelle génération épiscopale. $\mathrm{M}^{\mathrm{gr}}$ Tréhiou s'y impliqua pleinement et associa le sanctuaire de Sainte-Anne-d'Auray à cette dynamique en accueillant, après le dominicain Forestier et ses routiers à la pentecôte 1935, le congrès national de la PAC au moment des fêtes de Sainte-Anne. L'accent était clairement mis sur la paix, comme le signalait la présence de $\mathrm{M}^{\mathrm{gr}} \mathrm{Pic}$, l'évêque pacifiste de Gap, ainsi que le discours de l'abbé Bergey qui s'apprêtait à conduire ses anciens combattants dans les rues de Rome en septembre de la même année.

Fort de cette assise mobilisatrice maintenue, il fut décidé de mettre en scène la clôture des travaux, le 25 juillet 1937, de manière aussi spectaculaire qu'en 1932. Sans doute la fête a-t-elle moins marqué car elle ressemblait beaucoup à la précédente. Toutefois convient-il de souligner les capa-

56. Semaine religieuse du diocèse de Vannes, 5 août 1933, $\mathrm{n}^{\circ}$ 31, p. 500 . 
cités intactes de mobilisation de l'Église. Ce fait doit interroger l'historien car, tenté de l'inscrire dans une conjoncture européenne qui fut celle du déploiement de masse, il remarque que le catholicisme français - contrairement à ses voisins allemand ou belge - est longtemps resté étranger aux grands rassemblements. Le modèle irlandais semblerait d'ailleurs plus opérant sur le cas breton. Du moins peut-on souligner le recours aux recettes de mobilisation, comme cette double invitation, qui est une bonification par rapport à 1932 et pour pallier l'absence du nonce Valeri, du cardinal Verdier ${ }^{57}$ et du général Weygand. Ce dernier évoqua d'ailleurs dans son discours les « 240000 morts » de Bretagne. La symbolique mémorielle semblait ne jamais devoir s'épuiser puisque le moment fort de la journée a été constitué par le déploiement d'un grand drap mortuaire sur les escaliers du monument.

1937 n'est assurément pas le point final du mémorial qui reste encore un lieu à interroger pour les décennies qui suivent. On évoquera seulement, en s'en tenant aux perspectives de ce travail, que le régionalisme de Vichy représenté par ses préfets et donnant au mémorial une caution officielle, les $1^{\text {er }}$ novembre 1941 et 26 juillet 1942, constitue une étape de continuité et d'accentuation avec le passé décrit des années $1930{ }^{58}$. Dans un cadre différent, l'État continua d'ailleurs de dialoguer avec le monument en créant une nécropole nationale pour des combattants de la $2^{\mathrm{e}}$ Guerre mondiale près du sanctuaire. Cependant, le mémorial n'a pu dépasser son statut mémoriel lié à la Première Guerre mondiale et les actives tentatives des années 1970-1980 pour en faire un monument fédérateur de l'ensemble des guerres du xxe siècle ont été un échec (à l'exemple de ce gisant placé dans la crypte en 1974 puis mis en retrait à l'extérieur ou de cette flamme du souvenir restée à l'état de projet). Ainsi peut-on diagnostiquer un essoufflement de l'identification populaire et souligner d'importantes mutations. Au début des années 1970, l'Église contrainte à de coûteux travaux de restauration dut en appeler aux subventions publiques (celle de l'État et des 5 conseils généraux), devant la pauvreté d'une quête régionale ${ }^{59}$. Cependant, le mémorial vit toujours essentiellement en servant de cadre aux grandes célébrations du pèlerinage. Le lustre passé a même été ponctuellement égalé le 20 septembre 1996 lors de la venue de Jean-Paul II (avec une assistance comparable à celle de 1932). Certes, l'enceinte du monument, trop petite pour accueillir la foule du matin et la tribune de célébration, a été délaissée pour une prairie voisine et réservée à la célébration

57. En 1936, M ${ }^{\text {gr }}$ Liénart avait présidé les fêtes de Sainte-Anne, avant Gerlier en 1938 et Suhard en 1939.

58. Tout comme le pèlerinage politique du 26 juillet 1947 de Charles de Gaulle, alors chef du RPF, devant une assistance comparable à celle des années 1930 .

59. Les Archives de la basilique de Sainte-Anne-d'Auray révèlent ainsi que, malgré 8000 courriers envoyés par l'active association des Amis du mémorial créée en 1969, 2 paroisses du Finistère et 11 du Morbihan seulement ont répondu. Elles étaient 5 du diocèse de Saint-Brieuc pour un don de 230 francs. 
des familles plus restreinte de l'après-midi. Mais le souvenir des années vingt et trente a imprégné symboliquement la journée puisque l'on institua pour l'occasion un financement populaire de 20000 petits pavés (le " dallage de la foi ") posés sur une pelouse du sanctuaire. Vingt mille, c'est précisément le nombre d'inscriptions espérées sur le mur d'enceinte de 1914-1918. L'Église reste une institution de la mémoire.

Figure 1 - Dessin d'esquisse de l'architecte René Ménard (août 1922). À noter que le cortège est uniquement féminin (Arch. diocésaines de Vannes)

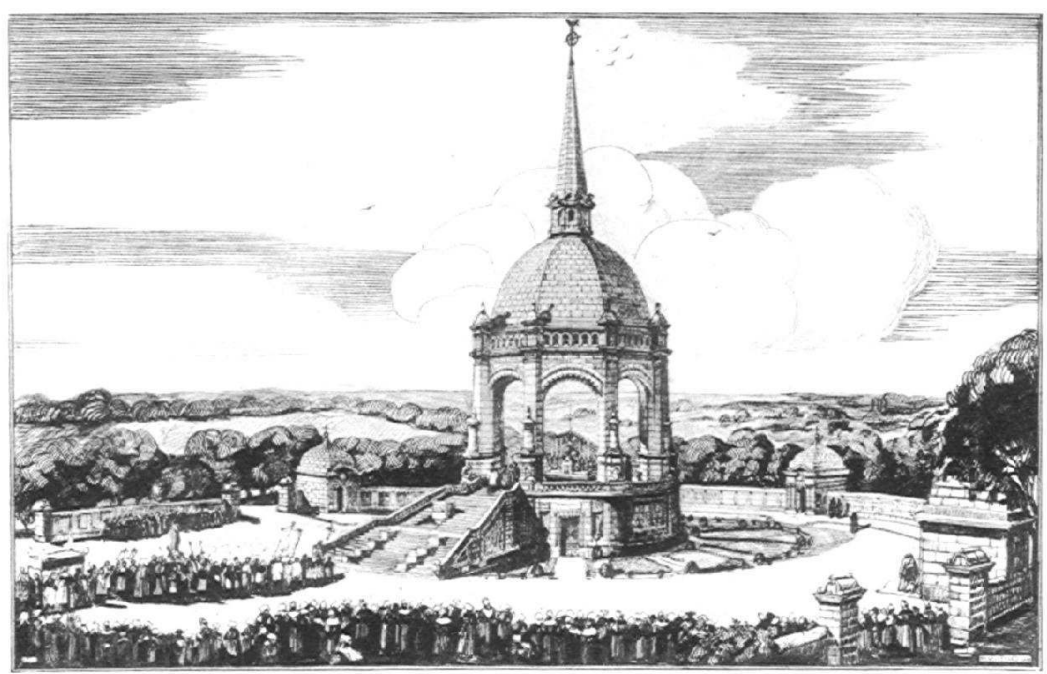

SAINTE-ANNE-DAURAY. -. Monument a la gloire des Bretons morts à la Guerre 
Figure 2-Le monument central aujourd'hui avec, sur la coupole, une croix différente de celle d'origine

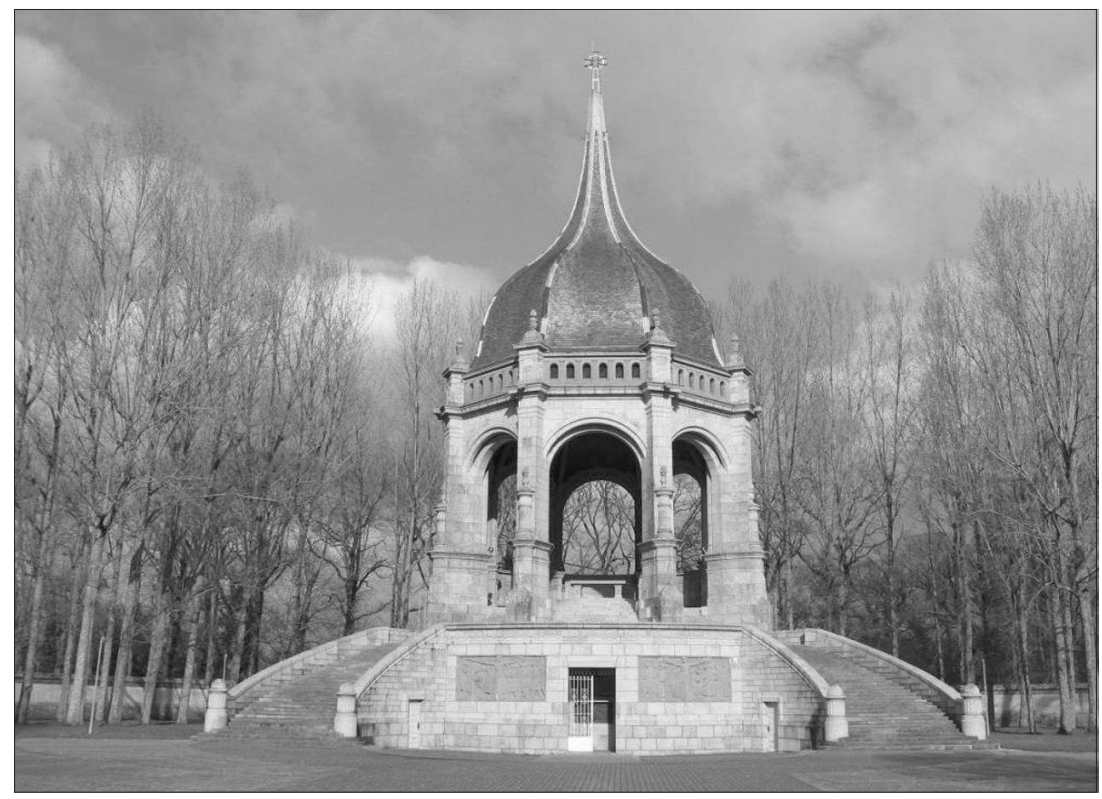

Figure 3 - Le cardinal Verdier assis au premier plan pendant le discours de $M^{\text {gr }}$ Duparc en juillet 1937

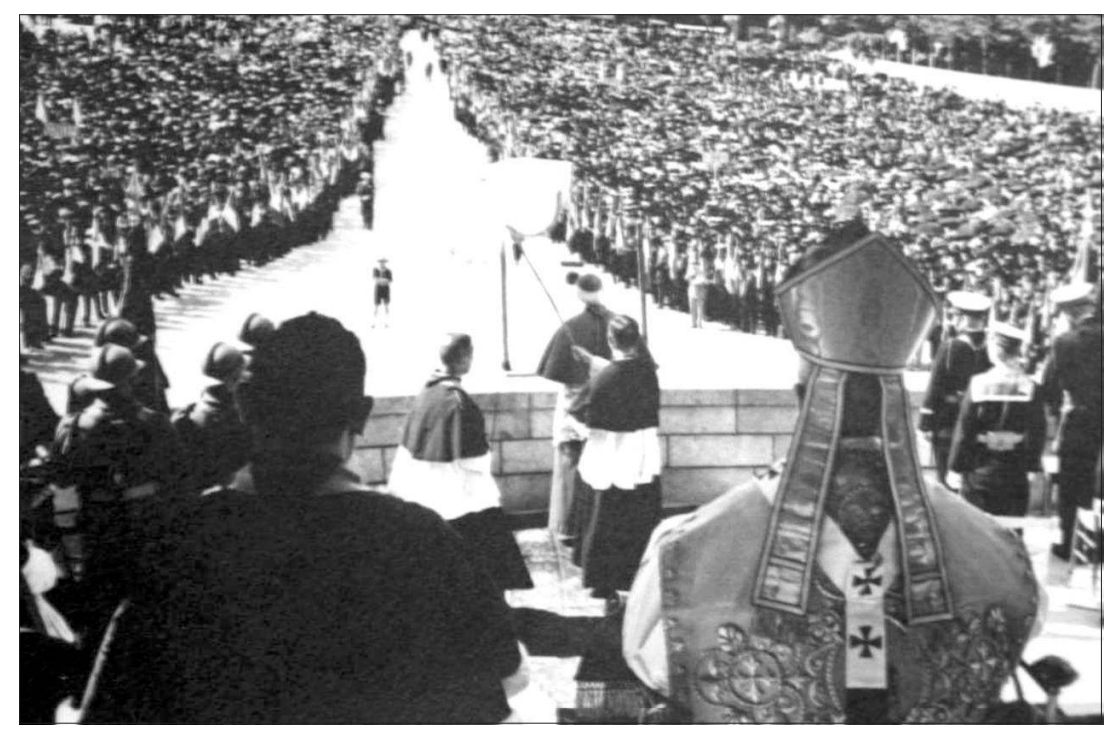


Figure 4 - Le rassemblement du 25 juillet 1937 (Arch. de la basilique de Sainte-Anne-d'Auray)

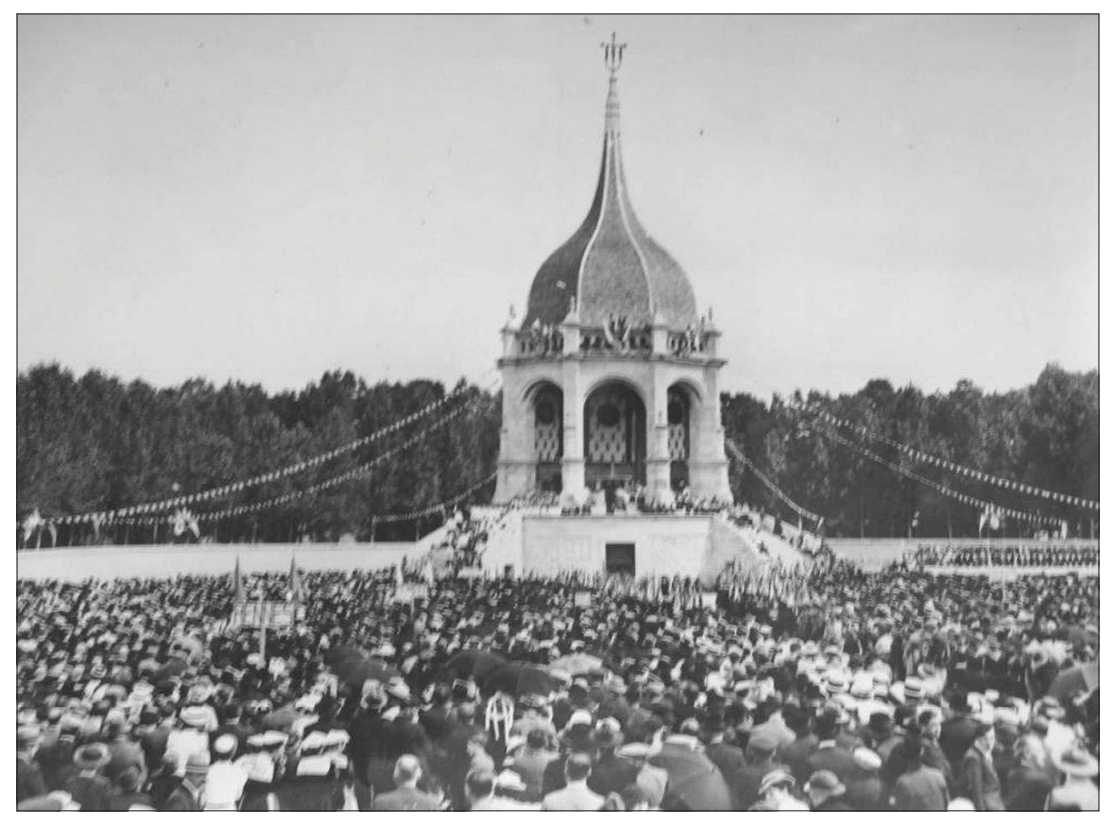




\section{RÉSUMÉ}

Le mémorial de Sainte-Anne-d'Auray peut-il être considéré comme le monument fédérateur de la mémoire de la Grande Guerre en Bretagne durant l'entre-deux-guerres? Non, si l'on regarde les conditions de sa création au début des années 1920 qui en font un monument très marqué de la défense catholique et qui a d'ailleurs eu du mal à s'imposer pour des raisons matérielles, financières et de déficit de reconnaissance du sanctuaire à l'échelle régionale. Oui cependant, si l'on considère que, dès la période du Cartel des gauches, le monument en cours d'élévation génère financement et adhésions populaires, grâce à une propagande habile, à la multiplication des rassemblements de masse et à l'absence de contre-projet. Les années 1930 constituent à ce titre une phase d'équilibre et d'achèvement. Le mémorial devient à la fois le sanctuaire du deuil collectif régional favorisant l'extension du pèlerinage voulue par la hiérarchie catholique et le théâtre d'impressionnantes manifestations fédératives d'anciens combattants en 1932 et 1937. Ce succès repose néanmoins sur l'ambiguïté de l'inflation sacrificielle qui en fait jusqu'à aujourd'hui le monument des " 240000 morts " de 1914-1918.

\section{ABSTRACT}

Can the Memorial at Sainte-Anne-d'Auray be considered as the monument that federated the memory of First World War in Britanny during the interwar years? The answer is no, with regard to the conditions of its creation in the early 1920s, which link it strongly to the defence of catholicism and which explain why it stood out so difficultly, for material and financial reasons and because of the lack of recognition of the sanctuary on the regional scale. But it can be viewed as a federative monument because, as soon as the Cartel des gauches period, the monument being erected aroused financing and popular adherence, thanks to a clever propaganda, the multiplication of mass gathering and the lack of counterplan. The 1930s constitute therefore a phase of balance and completion. The memorial became both the shrine of regional collective mourning - promoting thus the extension of pilgrimage wished by the catholic hierarchy - and the scene of impressive federative demonstrations of war veterans in 1932 and 1937. Success is nevertheless based on the ambiguity of sacrificial growth which makes it until now the monument of the "240,000 men killed" of the Great War 1914-1918. 